\title{
Experimental characterization of singlet scattering channels in long- range Rydberg molecules
}

\section{Journal Article}

Author(s):

Sassmannshausen, Heiner; Merkt, Frédéric; Deiglmayr, Johannes

Publication date:

2015-04-03

Permanent link:

https://doi.org/10.3929/ethz-a-010803700

Rights / license:

In Copyright - Non-Commercial Use Permitted

Originally published in:

Physical Review Letters 114(13), https://doi.org/10.1103/PhysRevLett.114.133201 
This article may be downloaded for personal use only. Any other use requires prior permission of the author and The American Physical Society (APS).

The following article appeared in Phys. Rev. Lett. 114, 133201 (2015) and may be found at https://doi.org/10.1103/PhysRevLett.114.133201. 


\title{
Experimental characterization of singlet scattering channels in long-range Rydberg molecules
}

\author{
Heiner Saßmannshausen, Frédéric Merkt, and Johannes Deiglmayr \\ Laboratory of Physical Chemistry, ETH Zurich, Switzerland*
}

\begin{abstract}
We observe the formation of long-range $\mathrm{Cs}_{2}$ Rydberg molecules consisting of a Rydberg and a ground-state atom by photoassociation spectroscopy in an ultracold Cs Rydberg gas near $6 \mathrm{~s}_{1 / 2}(F=3,4) \rightarrow n \mathrm{p}_{3 / 2}$ resonances $(n=26-34)$. The spectra reveal two types of molecular states recently predicted by D. A. Anderson, S. A. Miller, and G. Raithel [Phys. Rev. A 90, 062518 (2014)]: states bound purely by triplet $s$-wave scattering with binding energies ranging from $400 \mathrm{MHz}$ at $n=26$ to $80 \mathrm{MHz}$ at $n=34$, and states bound by mixed singlet-triplet $s$-wave scattering with smaller and $F$-dependent binding energies. The experimental observations are accounted for by an effective Hamiltonian including Fermi-contact $s$-wave scattering pseudopotentials, the hyperfine interaction of the ground-state atom, and the spin-orbit interaction of the Rydberg atom. The analysis enabled the characterization of the role of singlet scattering in the formation of long-range Rydberg molecules and the determination of an effective singlet $s$-wave scattering length for low-energy electron-Cs collisions.
\end{abstract}

Atoms in Rydberg states of high principal quantum number $n$ are weakly bound systems and are extremely sensitive to their environment. In 1934, Amaldi and Segrè [1] observed the pressure-dependent shift and broadening of the Rydberg series of alkali atoms in a gas cell, an effect which was explained by Fermi [2] as originating from the elastic scattering between slow Rydberg electrons and ground-state atoms within the Rydberg orbit. Specifically, he described the observed pressure shifts using a contact-type pseudopotential

$$
V(R)=2 \pi a|\Psi(\boldsymbol{R})|^{2},
$$

where $a$ is the scattering length and $|\Psi(\boldsymbol{R})|^{2}$ the probability density of the Rydberg electron at the position $\boldsymbol{R}$ of the neutral perturber. Measurements of pressure shifts in Rydberg states thus provide information on the cross sections of elastic collisions between slow electrons and atoms and molecules [2,3]. Equation (1) implies the existence of oscillating interaction potentials between Rydberg and ground-state atoms [4, 5]. A manifestation of such potentials are long-range diatomic molecules in which a ground-state atom having a negative $s$-wave scattering length is attached to a Rydberg atom at a distance corresponding to an antinode of $\Psi(\boldsymbol{R})$, as was first pointed out by Greene et al. [6]. Such molecules were first observed experimentally by Bendkowsky et al. [7] following excitation of ultracold $\mathrm{Rb}$ atoms close to $n \mathrm{~s}_{1 / 2}$ Rydberg states with $n=35-37$. Later investigations led to the detection of long-range $\mathrm{Rb}_{2}$ Rydberg molecules correlated to $n \mathrm{p}_{1 / 2,3 / 2}(n=7-12)[8], n \mathrm{~d}_{3 / 2,5 / 2} \quad(n=34-$ 40) [9], and $n \mathrm{~d}_{3 / 2,5 / 2}(n=40-49)$ [10] dissociation asymptotes and long-range $\mathrm{Cs}_{2}$ Rydberg molecules correlated to $n \mathrm{~s}_{1 / 2}(n=31-34)[11]$ and $n \mathrm{~s}_{1 / 2}(n=37,39,40)$ [12] dissociation asymptotes. The analysis of the experimental data confirmed the overall validity of Eq. (1), revealed important contributions arising from triplet $p$-wave scattering channels, and enabled the determination of triplet $s$ - and $p$-wave scattering lengths that confirmed theoret- ical predictions [13].

Singlet $s$-wave scattering lengths are expected to be either positive or much smaller than triplet scattering lengths [14] and thus scattering in singlet channels has not been included in the analysis of the experimental data. In a very insightful article, Anderson et al. [15] have recently predicted that the hyperfine coupling in the ground-state atom should effectively mix triplet and singlet scattering channels and lead to shallow bound states in addition to the more strongly bound states resulting from pure triplet scattering. These states would offer the possibility to obtain experimental information on singlet $s$ - and $p$-wave scattering lengths which, so far, have only been predicted theoretically $[14,16]$.

Here, we report on the observation of these shallow bound states correlated to $n \mathrm{p}_{3 / 2}$ Rydberg states in the range $n=26-34$ in an ultracold Cs gas. We show that the well depths of these shallow states depend on the hyperfine component $(F=3$ or $F=4)$ in which the ground-state atoms are prepared, model the experimental observations theoretically, and extract an effective singlet $s$-wave scattering length for low-energy $e^{-}$-Cs collisions from the experimental data.

Following Ref. [15], we model the interaction between the Rydberg and the ground-state atom using

$$
\hat{H}=\hat{H}_{0}+\hat{H}_{\mathrm{SO}}+\hat{H}_{\mathrm{HF}}+\hat{P}_{\mathrm{S}} \cdot \hat{V}_{\mathrm{S}}+\hat{P}_{\mathrm{T}} \cdot \hat{V}_{\mathrm{T}},
$$

where $\hat{H}_{0}$ is the Hamiltonian of the Rydberg atom without spin-orbit interaction, $\hat{H}_{\mathrm{SO}}=A_{\mathrm{SO}} \boldsymbol{\ell}_{r} \otimes \boldsymbol{s}_{r}$ is the spin-orbit interaction of the Rydberg electron with angular momentum $\boldsymbol{\ell}_{r}$ and electron spin $\boldsymbol{s}_{r}$, and $\hat{H}_{\mathrm{HF}}=$ $A_{\mathrm{HF}} \boldsymbol{i}_{g} \otimes \boldsymbol{s}_{g}$ is the hyperfine interaction of the groundstate atom with nuclear spin $\boldsymbol{i}_{g}$ and electron spin $\boldsymbol{s}_{g}$. Separate terms for singlet $(\mathrm{S})$ and triplet $(\mathrm{T})$ scattering are obtained by using the projection operators $\hat{P}_{\mathrm{S}}=$ $-\boldsymbol{s}_{r} \otimes \boldsymbol{s}_{g}+\frac{1}{4} \cdot \hat{\mathbb{1}}$ and $\hat{P}_{\mathrm{T}}=\boldsymbol{s}_{r} \otimes \boldsymbol{s}_{g}+\frac{3}{4} \cdot \hat{\mathbb{1}}$ and representing the corresponding Fermi-contact-interaction operators by $\hat{V}_{i}=2 \pi a_{i} \hat{\delta}^{3}(\boldsymbol{r}-R \hat{\boldsymbol{z}})$, with $i=\mathrm{S}$, T, for $s$-wave 


\begin{tabular}{|cc|c|c|c|c|}
\hline \multicolumn{2}{|c|}{ Rydberg asymptote } & Contributions & $g_{3}$ & $g_{4}$ & Binding via \\
\hline \multirow{2}{*}{$n \mathrm{p}_{1 / 2}$} & \multirow{2}{*}{$\left|\omega_{r}\right|=1 / 2$} & ${ }^{1} \Sigma^{+},{ }^{3} \Sigma^{+},{ }^{1} \Pi,{ }^{3} \Pi$ & 8 & 8 & $a_{\mathrm{S}}, a_{\mathrm{T}}$ \\
\cline { 3 - 6 } & & ${ }^{3} \Sigma^{+},{ }^{1} \Pi,{ }^{3} \Pi$ & 6 & 10 & $a_{\mathrm{T}}$ \\
\hline \multirow{2}{*}{$n \mathrm{p}_{3 / 2}$} & \multirow{2}{*}{$\omega_{r} \mid=1 / 2$} & ${ }^{1} \Sigma^{+},{ }^{3} \Sigma^{+},{ }^{1} \Pi,{ }^{3} \Pi$ & 8 & 8 & $a_{\mathrm{S}}, a_{\mathrm{T}}$ \\
\cline { 2 - 7 } & ${ }^{3} \Sigma^{+},{ }^{1} \Pi,{ }^{3} \Pi$ & 6 & 10 & $a_{\mathrm{T}}$ \\
\cline { 2 - 7 } & $\left|\omega_{r}\right|=3 / 2$ & ${ }^{1} \Pi,{ }^{3} \Pi$ & 14 & 18 & - \\
\hline
\end{tabular}

TABLE I. Asymptotes and binding mechanism. First column: fine-structure asymptote of the Rydberg atom. Second column: symmetries contributing to the molecular state. Third and fourth column: degeneracies $g_{3}$ and $g_{4}$ of the molecular states associated with $n \mathrm{p}_{j}, 6 \mathrm{~s}_{1 / 2}(F=3,4)$ asymptotes. Last column: $s$-wave scattering lengths contributing to the binding.

scattering between the Rydberg electron at position $\boldsymbol{r}$ and the ground-state atom located at distance $R$ along the molecular $z$-axis [17]. We neglect the rotational motion of the molecule, because the spacings between the accessible rotational levels are less than their natural linewidths. The hyperfine structure of the Rydberg atom is also neglected, because the corresponding interaction is much weaker than all other relevant interactions [18]. To reproduce experimental binding energies, we adjust the zero-energy limit of the energy-dependent scattering lengths (Ref. [14], Eqs. (1,4-6)). The spin-orbit coupling constants $A_{\mathrm{SO}}$ are extracted from experimental fine-structure splittings [19] and we use the hyperfine coupling constant $A_{\mathrm{HFS}}$ from Ref. [20].

The Hamiltonian matrix corresponding to Eq. (2) is set up in the uncoupled basis $\left|\ell_{r}, \lambda_{r}\right\rangle\left|s_{r}, \sigma_{r}\right\rangle\left|s_{g}, \sigma_{g}\right\rangle\left|i_{g}, \omega_{i, g}\right\rangle$ $\left(i_{g}=7 / 2\right.$ for $\left.{ }^{133} \mathrm{Cs}\right)$ for a single $n \mathrm{p}$ Rydberg state and the energetically closest Rydberg state, $(n-1)$ d. The quantum number $\Omega_{\text {tot }} \equiv \lambda_{r}+\sigma_{r}+\sigma_{g}+\omega_{i, g}$ of the projection of the total angular momentum on the internuclear axis is a good quantum number. The ground-state atom can be in either of the two $F=3,4\left(\boldsymbol{F} \equiv \boldsymbol{i}_{g}+\boldsymbol{s}_{g}\right)$ hyperfine levels, and the $n$ p Rydberg atom can be in either the $n \mathrm{p}_{3 / 2}\left(\omega_{r} \equiv \lambda_{r}+\sigma_{r}=-3 / 2,-1 / 2,1 / 2,3 / 2\right)$ or the $n \mathrm{p}_{1 / 2}$ $\left(\omega_{r}=-1 / 2,1 / 2\right)$ spin-orbit levels. The spin-orbit interaction of the Rydberg electron completely mixes the $\lambda_{r}=0$ and $\left|\lambda_{r}\right|=1$ components of the $\left|\omega_{r}\right|=1 / 2$ levels, whereas the $\left|\omega_{r}\right|=3 / 2$ levels have pure $\Pi$ character. Because we restrict the $e^{-}$-Cs interaction to $s$-wave scattering, only the $\lambda_{r}=0\left(\Sigma^{+}\right)$components of the Rydberg wavefunction contribute to the binding [17]. The $\left|\omega_{r}\right|=3 / 2$ levels are thus non-bonding and the strength of the bond of the $\left|\omega_{r}\right|=1 / 2$ levels is proportional to their $\lambda_{r}=0\left(\Sigma^{+}\right)$ character. The selection of the $\lambda_{r}=0$ component in the interaction terms $\hat{V}_{\mathrm{S}}$ and $\hat{V}_{\mathrm{T}}$ implies that $\omega_{r}$ equals $\sigma_{r}$ in all components of the wavefunctions contributing to binding and that $s$-wave singlet and triplet channels of the $e^{-}$-Cs scattering interaction are not mixed by $\hat{H}_{\mathrm{SO}}$. These channels can, however, be mixed by $\hat{H}_{\mathrm{HF}}$. Remarkably, the eigenstates of Eq. (2) form two distinct subgroups, one containing states which do not possess any ${ }^{1} \Sigma^{+}$character (but ${ }^{3} \Sigma^{+},{ }^{1} \Pi$ and ${ }^{3} \Pi$ ) and for which the binding is entirely dictated by the triplet $s$-wave interaction, and one containing states which possess both ${ }^{3} \Sigma^{+}$and ${ }^{1} \Sigma^{+}$(as well as ${ }^{1} \Pi$ and ${ }^{3} \Pi$ ) character and for which the binding results from both singlet and triplet $s$-wave scattering interactions. Table I gives an overview of the character and degeneracy factors of the resulting bound states. For simplicity we refer in the following to the states bound via the ${ }^{3} \Sigma^{+}$component as ${ }^{3} \Sigma$ states and to those for which the binding results from both ${ }^{3} \Sigma^{+}$and ${ }^{1} \Sigma^{+}$components as ${ }^{1,3} \Sigma$ states. The ${ }^{3} \Sigma$ states are those that have been observed in previous experiments [7-9].

A Cooper minimum in the $6 \mathrm{~s}_{1 / 2} \rightarrow n \mathrm{p}_{1 / 2}$ photoexcitation cross section of Cs [21] prevents the observation of states correlated to $n \mathrm{p}_{1 / 2}$ asymptotes and we thus focus on the states correlated to $n \mathrm{p}_{3 / 2}$ asymptotes. Potentialenergy curves (PECs) for the long-range molecules are obtained by determining the eigenvalues of Eq. (2) as a function of the separation $R$ between the atoms. We use the mapped Fourier-Grid method [22] to obtain the vibronic eigenstates of these PECs. The PECs and vibrational wavefunctions of the $26 \mathrm{p}_{3 / 2},{ }^{3} \Sigma$ and $26 \mathrm{p}_{3 / 2},{ }^{1,3} \Sigma$ states, calculated for parameters derived from the analysis of our spectroscopic data, are depicted in Fig. 1. The ${ }^{3} \Sigma$ PECs do not depend on the hyperfine state of the ground-state atom, while the ${ }^{1,3} \Sigma$ state correlated to the $F=3$ asymptote is more strongly bound than the ${ }^{1,3} \Sigma$ state correlated to the $F=4$ asymptote.

The long-range $\mathrm{Cs}_{2}$ molecules are photoassociated from samples of ultracold Cs atoms $\left(3 \cdot 10^{8}\right.$ atoms at a density of $2 \cdot 10^{11} \mathrm{~cm}^{-3}$ and a temperature of $\left.40 \mu \mathrm{K}\right)$, re-
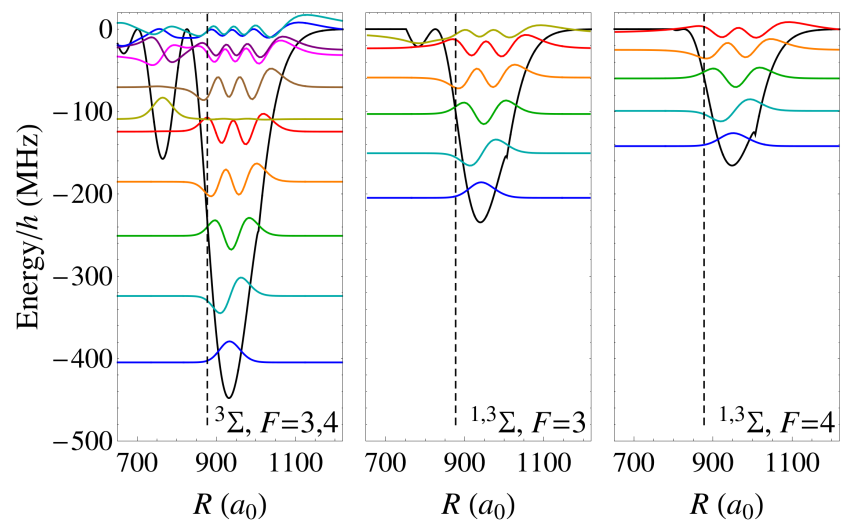

FIG. 1. (Color online) Potential-energy curves (black lines) and vibrational wave functions (colored lines) for ${ }^{3} \Sigma$ states dissociating to the $26 p_{3 / 2}, 6 \mathrm{~s}_{1 / 2}(F=3,4)$ asymptotes (left panel), ${ }^{1,3} \Sigma$ state dissociating to the $26 p_{3 / 2}, 6 \mathrm{~s}_{1 / 2}(F=3)$ (middle panel), and $26 p_{3 / 2}, 6 \mathrm{~s}_{1 / 2}(F=4)$ (right panel) asymptote. Vertical dashed lines mark the positions where the semiclassical Rydberg-electron energy equals the energy of the ${ }^{3} P_{0}$ Cs- $e^{-}$scattering resonance (see text). 

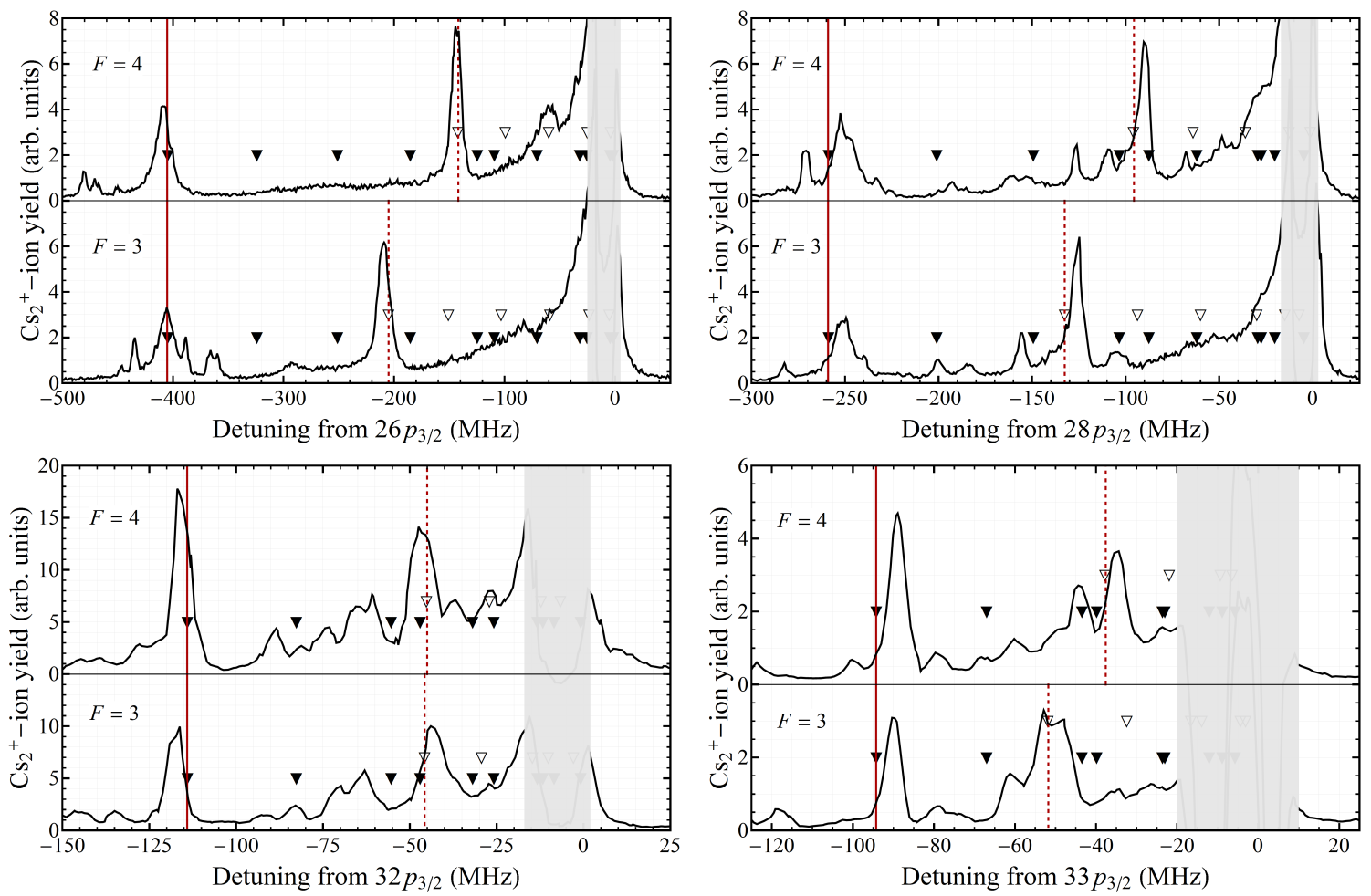

FIG. 2. Detected $\mathrm{Cs}_{2}^{+}$ions as function of laser frequency near the $n \mathrm{p}_{3 / 2}, 6 \mathrm{~s}_{1 / 2}(F=3,4)$ asymptotes. Solid (dashed) red lines mark the calculated positions of the $v=0$ levels of ${ }^{3} \Sigma\left({ }^{1,3} \Sigma\right)$ states. Calculated positions of higher vibrational levels are also shown (full and open triangles, respectively). The strongly saturated atomic transitions correspond to the grey areas.

leased from a vapor-loaded compressed magneto-optical trap (cMOT) [23][24]. After $10 \mathrm{~ms}$ of compression, the magnetic field of the cMOT is switched off and the atoms are cooled during an optical-molasses phase. After $5 \mathrm{~ms}$, magnetic fields caused by eddy currents have decayed and the residual magnetic fields (after compensation) are on the order of few $\mathrm{mG}$ [18]. The atoms are then optically pumped into one of the two hyperfine states $(F=3,4)$ of the $6 \mathrm{~s}_{1 / 2}$ ground state and excited with a single-mode cw UV laser (Coherent 899-21 ring dye laser with Coherent MBD200 external doubling unit, $100 \mathrm{~mW}$ UV power, $5 \mathrm{MHz}$ linewidth, $40 \mu$ s excitation pulse length) using intensities around $100 \mathrm{~W} / \mathrm{cm}^{2}$. The frequency relative to the $n \mathrm{p}_{3 / 2}$ atomic resonance frequency is calibrated at an accuracy of $5 \mathrm{MHz}$ using a scanning Fabry-Perot cavity and a frequency-stabilized HeNe laser. After excitation, spontaneously formed ions [25] are detected with a multichannel-plate (MCP) detector. On molecular resonances, we observe both atomic $\mathrm{Cs}^{+}$(attributed to Penning ionization) and molecular $\mathrm{Cs}_{2}^{+}$ions (attributed to associative ionization), the relative strength of the former increasing with the density of excited molecules.

Fig. 2 presents photoassociation spectra of Cs atoms prepared in the $F=3$ and $F=4$ hyperfine levels to $\mathrm{Cs}_{2}$ Rydberg molecules correlated to $n \mathrm{p}_{3 / 2}$ Rydberg states $(n=26,28,32$, and 33). Several photoassociation res- onances are observed on the low-frequency side of the strongly saturated atomic $6 \mathrm{~s}_{1 / 2}(F=3,4) \rightarrow n \mathrm{p}_{3 / 2}$ resonances, marked by grey areas. The two most intense photoassociation resonances are assigned to the vibronic ground state of the ${ }^{3} \Sigma$ and ${ }^{1,3} \Sigma$ states. In the same manner, we determine the binding energies of the ${ }^{3} \Sigma(v=0)$ and ${ }^{1,3} \Sigma(v=0)$ levels for all $n$ values between 26 and 34. In Fig. 3 these energies are compared to binding energies calculated with the model outlined above after adjustment of the zero-energy $s$-wave singlet $\left(a_{\mathrm{S}, 0}\right)$ and triplet $\left(a_{\mathrm{T}, 0}\right)$ scattering lengths, to $a_{\mathrm{T}, 0}=-21.8 \pm 0.2 a_{0}$ and $a_{\mathrm{S}, 0}=-3.5 \pm 0.4 a_{0}$, respectively, in a leastsquares fit [26]. The fact that the adjustment of only two parameters permits the reproduction of 36 resonances within their experimental uncertainty of $5 \mathrm{MHz}$ leaves no doubt concerning the validity of the assignments. As predicted by the model calculations (see also Fig. 1), the positions of the ${ }^{3} \Sigma(v=0)$ levels are identical for $F=3$ and $F=4$ within the experimental uncertainty whereas $(F=4),{ }^{1,3} \Sigma(v=0)$ levels are less strongly bound than the $(F=3),{ }^{1,3} \Sigma(v=0)$ levels. An exception is found at $n=32$, where the ${ }^{1,3} \Sigma(v=0)$ levels correlated to $32 \mathrm{p}_{3 / 2}, 6 \mathrm{~s}_{1 / 2}(F=3)$ and $32 \mathrm{p}_{3 / 2}, 6 \mathrm{~s}_{1 / 2}(F=4)$ have almost identical binding energies. This irregularity is well described by our model, which indicates that it originates from an accidental perturbation of the $32 \mathrm{p}_{3 / 2}, 6 \mathrm{~s}_{1 / 2}(F=3)$ 


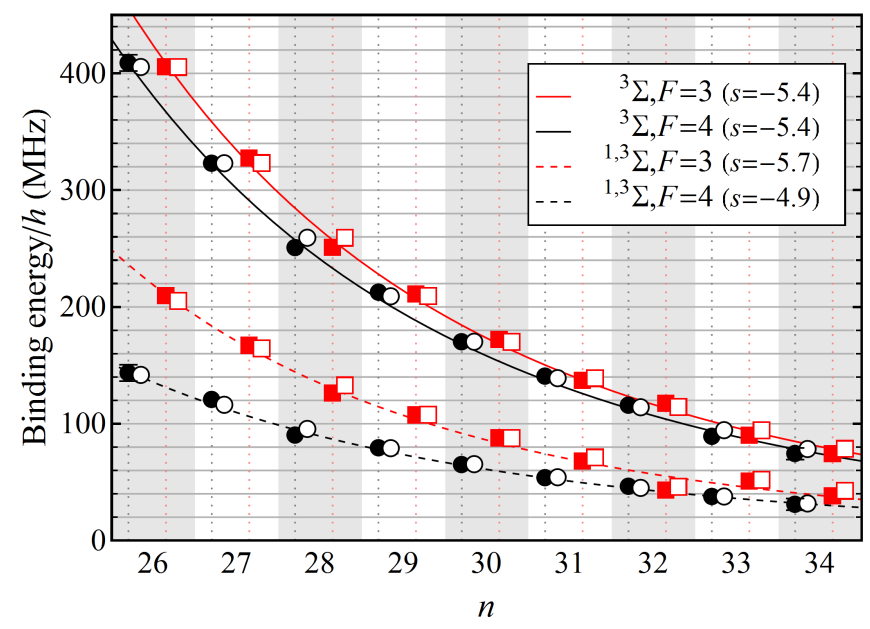

FIG. 3. Comparison of experimental (full symbols) and calculated (open symbols) binding energies of the ${ }^{3} \Sigma(v=0)$ and ${ }^{1,3} \Sigma(v=0)$ levels. The solid/dashed lines are fits of a $\left(n^{*}\right)^{s}$ scaling law to the experimental binding energies to guide the eye. Black and red symbols designate states with the groundstate atoms prepared in $F=4$ and $F=3$, respectively.

state by the almost degenerate $32 \mathrm{p}_{1 / 2}, 6 \mathrm{~s}_{1 / 2}(F=4)$ state. Indeed, at $n=32$, the $n \mathrm{p}_{1 / 2}-n \mathrm{p}_{3 / 2}$ fine-structure splitting, which scales as $n^{*-3}$, almost exactly matches the ground-state hyperfine splitting. The dependence of the ${ }^{3} \Sigma(v=0)$ binding energies on the effective quantum number $n^{*}$ deviates from the previously encountered $n^{*-6}$ scaling [7] (see Fig. 3). This deviation is also present in the calculated binding energies and is caused by the increasing contribution of higher-order, energy-dependent terms of the scattering length for lower $n$.

Our values of the triplet and singlet $s$-wave scattering lengths are effective, model-based values obtained assuming that $p$-wave scattering is negligible. This assumption is fulfilled for low electron energies and in the absence of scattering resonances. The lowest $p$-wave scattering resonance (the ${ }^{3} P_{0}$ shape resonance in $\mathrm{Cs}^{-}[27]$ ) is located at an electron kinetic energy $\frac{p_{c}^{2}}{2 m_{e}}$ of $4 \mathrm{meV}$ [11] which is (in a semi-classical description) reached by the Rydberg electron at the critical radius $r_{c}$ corresponding to $p_{c}^{2}=\left(-\frac{1}{n^{* 2}}+\frac{2}{r_{c}}\right)$, and marked in Fig. 1 for $n=26$ by vertical dashed lines. Although the minima of the outermost potential wells are located beyond $r_{c}$ and the $v=0$ levels should not be strongly affected by $p$-wave scattering for $n \geq 26$, Fig. 1 also indicates that PECs for lower $n$ values and excited vibrational levels of the outermost well could be significantly impacted by $p$-wave scattering channels. We believe this to be the reason for the inability of our effective $s$-wave scattering model to reliably predict the positions of excited vibrational levels, which are marked by triangles in Fig. 2, and of further resonances observed in our experiments for $n=19-25$. A similar observation was made in studies of long-range $n$ s Rydberg molecules in $\mathrm{Rb}$ where pure triplet $s$-wave scattering was sufficient to describe the observed ${ }^{3} \Sigma(v=0)$ binding energies [7], but a more complete model, including $p$-wave scattering and quantum reflection at short range, was required to reproduce the positions of excited vibrational levels [13].

Fabrikant [14] and, more recently, Bahrim et al. [16] calculated the $e^{-}$-Cs $s$-wave scattering lengths to be $a_{\mathrm{T}, 0}=-22.7 \quad a_{0}$ and $a_{\mathrm{S}, 0}=-2.4 \quad a_{0}$, and $a_{\mathrm{T}, 0}=-21.7 \quad a_{0}$ and $a_{\mathrm{S}, 0}=-1.33 a_{0}$, respectively, from an effective-rangetheory analysis of higher-energy scattering data. As appropriate measure of the deviation between experiment and theory, we consider the absolute error in the zero-energy scattering phase shift $\delta_{0}=\arctan \left(a_{i} / R_{\mathrm{pol}}\right)+$ $\pi / 4$ [28], where $R_{\mathrm{pol}}=\sqrt{\alpha}=20.05 a_{0}$ is the typical range of the $e^{-}$-Cs interaction and $\alpha$ is the polarizability of the Cs ground-state atom. The deviation between experiment and theory is at most 0.1 radian and the agreement can thus be regarded as good. Our fitted model-based values may include effects of higher partial waves in the $e^{-}$Cs scattering and interactions with Rydberg states other than the neighboring $(n-1) \mathrm{d}_{3 / 2,5 / 2}$ states in an effective manner. The fact that our model reproduces the experimental observations well over a broad range of $n$ from 26 to 34 suggests that these effects are not dominant.

The interaction operators $\hat{V}_{i}$ in Eq. (2) mix the neighboring $n \mathrm{p}$ and $(n-1) \mathrm{d}$ Rydberg states and induce an electric dipole moment in the molecular frame $[9$, 11, 29]. For the $32 \mathrm{p}_{3 / 2}, 6 \mathrm{~s}_{1 / 2}(F=4),{ }^{3} \Sigma(v=0)$ states, our model predicts dipole moments ranging from 21.0 to $25.8 \mathrm{D}$ (mean value $24.4 \mathrm{D}$ ) for $\left|\Omega_{\text {tot }}\right|$ values between $1 / 2$ and $9 / 2$. The dipole moments predicted for the $32 \mathrm{p}_{3 / 2}, 6 \mathrm{~s}_{1 / 2}(F=4),{ }^{1,3} \Sigma(v=0)$ states are smaller and range from -0.5 to $12.0 \mathrm{D}$ (mean value $5.6 \mathrm{D}$ ) for $\left|\Omega_{\mathrm{tot}}\right|$ values between $1 / 2$ and $7 / 2$. In an electric field we observe broadenings for these states which are in qualitative agreement with the expected Stark shifts, however the spectra also reveal the strong perturbations due to field-induced state mixing [30] as observed in long-range $\mathrm{Rb}_{2}$ dimers [29].

The prominent contributions of the long-range molecules in ${ }^{1,3} \Sigma$ states to the photoassociation spectrum of Cs near the $n \mathrm{p}_{3 / 2}, 6 \mathrm{~s}_{1 / 2}$ asymptotes implies that these states should also be observable in other systems. In Refs. [7, 13], long-range $\mathrm{Rb}_{2}$ Rydberg molecules were studied by photoassociation of an ultracold $\mathrm{Rb}$ sample prepared in the $5 \mathrm{~s}_{1 / 2}\left(F=2, m_{F}=2\right)$ level in a magnetic trap. Thus, only the shallow ${ }^{1,3} \Sigma$ states correlating to the $n \mathrm{~s}_{1 / 2}, 5 \mathrm{~s}_{1 / 2}(F=2)$ asymptotes could have been observed. Using a triplet $s$-wave scattering length of $a_{\mathrm{T}, 0}=-18.5 a_{0}[7]$ and the literature value for $a_{\mathrm{S}, 0}=$ $+0.627 a_{0}$ [16] we predict the positions of the $v=0$ levels of the $n=35-37^{1,3} \Sigma$ shallow potential wells listed in the column $E_{2,1,3 \Sigma}^{\mathrm{Th}}$ of Tab. II. Near these positions, all spectra presented in Fig. 2 of Ref. [13] show features marked by dashed lines at positions given in the column $E_{2, \dagger}^{\operatorname{Exp}}$ of Tab. II. After a small adjustment of 


\begin{tabular}{|c|c|c||c|c||c|c|}
\hline$n$ & $E_{2,3 \Sigma}^{\mathrm{Exp}}$ & $E_{2, \dagger}^{\mathrm{Exp}}$ & $E_{2,{ }^{3} \Sigma}^{\mathrm{Th}}$ & $E_{2,1,3 \Sigma}^{\mathrm{Th}}$ & $E_{2,1,3 \Sigma}^{*}$ & $E_{1,1,3 \Sigma}^{*}$ \\
\hline 35 & -23.0 & -3.8 & -23.1 & -3.9 & -3.6 & -11.0 \\
\hline 36 & -19.0 & -2.9 & -19.2 & -3.2 & -2.9 & -9.1 \\
\hline 37 & -16.2 & -2.3 & -16.0 & -2.6 & -2.4 & -7.5 \\
\hline
\end{tabular}

TABLE II. Experimental positions $E_{2}^{\text {Exp }}$ of the ${ }^{3} \Sigma(v=0)$ resonances correlated to ${ }^{87} \mathrm{Rb}, n \mathrm{~s}_{1 / 2}, 5 \mathrm{~s}_{1 / 2}(F=2)$ asymptotes from Ref. [13], and calculated positions $E^{\mathrm{Th}}$ of $v=0$ levels in ${ }^{3} \Sigma$ and ${ }^{1,3} \Sigma$ states. Also given are $v=0$ binding energies $E_{F, 1,3 \Sigma}^{*}$ for $F=1,2$ based on optimized values $a_{\mathrm{T}, 0}=-18.7 a_{0}$ and $a_{\mathrm{S}, 0}=+1.0 a_{0}$. All values in $\mathrm{MHz}$.

$a_{\mathrm{S}, 0}$ to $1.0 a_{0}$, our calculations of the positions of the ${ }^{1,3} \Sigma(v=0)$ levels for $F=2$ match all experimental peak positions within $0.2 \mathrm{MHz}$ (see column $E_{2,1,3 \Sigma}^{*}$ in Tab. II). In Ref. [13], these resonances on the low-frequency side of the atomic transitions were, however, attributed to a purely atomic transition, the shift being the electron-spin Zeeman shift of the $n \mathrm{~s}_{1 / 2}$ Rydberg level. Our calculations for the ${ }^{1,3} \Sigma(v=0)$ levels associated with the $F=1$ component of the ground-state level predict larger binding energies (see column $E_{1,1,3 \Sigma}^{*}$ in Tab. II) and we hope that our predictions will be confirmed by future experiments with ultracold $\mathrm{Rb}$ samples prepared in the $F=1$ hyperfine component.

In previous studies of ${ }^{3} \Sigma$ long-range Rydberg $\mathrm{Rb}$ molecules correlated to $n \mathrm{~s}_{1 / 2}$ Rydberg states, not only dimers, but also trimers and even larger $\mathrm{Rb}_{m}$ molecules have been observed [31], the binding energies being proportional to the number $(m-1)$ of ground-state atoms. We have not observed any long-range molecules consisting of more than two atoms. We attribute this nonobservation to a reduction of the excitation probability of $m=3$ long-range Rydberg molecules by a factor of $(4 \pi)^{-1}$ resulting from the change from spherical to linear geometry (imposed by the anisotropy of the $p$-orbitals) when going from $n$ s Rydberg levels to $n$ p Rydberg levels.

This work is supported financially by the Swiss National Science Foundation under Project Nr. 200020146759, and the NCCR QSIT, and the EU Initial Training Network COHERENCE under grant FP7-PEOPLE2010-ITN-265031.

* jdeiglma@ethz.ch

[1] E. Amaldi and E. Segrè, Il Nuovo Cimento 11, 145 (1934).

[2] E. Fermi, Il Nuovo Cimento 11, 157 (1934).

[3] H. Massey and E. Burhop, Electronic and Ionic impact phenomena, Vol. 1 (Clarendon Press, Oxford, 1969).

[4] G. Vitrant, J. M. Raimond, M. Gross, and S. Haroche, J. Phys. B 15, L49 (1982).

[5] E. de Prunelé, Phys. Rev. A 35, 496 (1987).

[6] C. H. Greene, A. S. Dickinson, and H. R. Sadeghpour, Phys. Rev. Lett. 85, 2458 (2000).
[7] V. Bendkowsky, B. Butscher, J. Nipper, J. P. Shaffer, R. Löw, and T. Pfau, Nature 458, 1005 (2009).

[8] M. A. Bellos, R. Carollo, J. Banerjee, E. E. Eyler, P. L. Gould, and W. C. Stwalley, Phys. Rev. Lett. 111, 053001 (2013).

[9] D. A. Anderson, S. A. Miller, and G. Raithel, Phys. Rev. Lett. 112, 163201 (2014).

[10] A. T. Krupp, A. Gaj, J. B. Balewski, P. Ilzhöfer, S. Hofferberth, R. Löw, T. Pfau, M. Kurz, and P. Schmelcher, Phys. Rev. Lett. 112, 143008 (2014).

[11] J. Tallant, S. T. Rittenhouse, D. Booth, H. R. Sadeghpour, and J. P. Shaffer, Phys. Rev. Lett. 109, 173202 (2012).

[12] D. Booth, S. Rittenhouse, J. Yang, H. Sadeghpour, and J. Shaffer, arXiv:1411.5291 (2014).

[13] V. Bendkowsky, B. Butscher, J. Nipper, J. B. Balewski, J. P. Shaffer, R. Löw, T. Pfau, W. Li, J. Stanojevic, T. Pohl, and J. M. Rost, Phys. Rev. Lett. 105, 163201 (2010).

[14] I. I. Fabrikant, J. Phys. B 19, 1527 (1986).

[15] D. A. Anderson, S. A. Miller, and G. Raithel, Phys. Rev. A 90, 062518 (2014).

[16] C. Bahrim, U. Thumm, and I. I. Fabrikant, J. Phys. B 34, L195 (2001).

[17] A. Omont, J. Phys. (Paris) 38, 1343 (1977).

[18] H. Saßmannshausen, F. Merkt, and J. Deiglmayr, Phys. Rev. A 87, 032519 (2013).

[19] P. Goy, J. M. Raimond, G. Vitrant, and S. Haroche, Phys. Rev. A 26, 2733 (1982).

[20] E. Arimondo, M. Inguscio, and P. Violino, Rev. Mod. Phys. 49, 31 (1977).

[21] J. M. Raimond, M. Gross, C. Fabre, S. Haroche, and H. H. Stroke, J. Phys. B 11, L765 (1978).

[22] V. Kokoouline, O. Dulieu, R. Kosloff, and F. MasnouSeeuws, J. Chem. Phys. 110, 9865 (1999).

[23] W. Petrich, M. H. Anderson, J. R. Ensher, and E. A. Cornell, J. Opt. Soc. B 11, 1332 (1994).

[24] We achieve higher densities of $2 \cdot 10^{12} \mathrm{~cm}^{-3}$ in our setup using a crossed far-off-resonance optical dipole trap (FORT) [32]. However the signal loss due to the reduction in atom number is not compensated by the increased density.

[25] Comparable spectra are obtained when using field ionization and detection of $\mathrm{Cs}^{+}$ions.

[26] If we model the energy dependence of the scattering lengths by $a(k)=a_{0}+\frac{\pi}{3} \alpha k(R)$ with the ground-state atom polarizability $\alpha=402.2$ a.u. and the semiclassical electron momentum $k=\sqrt{2 / R-1 / n^{* 2}}$ [17], we find $a_{\mathrm{T}, 0}=-20.5 \pm 0.2 a_{0}$ and $a_{\mathrm{S}, 0}=-3.7 \pm 0.4 a_{0}$.

[27] M. Scheer, J. Thøgersen, R. C. Bilodeau, C. A. Brodie, H. K. Haugen, H. H. Andersen, P. Kristensen, and T. Andersen, Phys. Rev. Lett. 80, 684 (1998).

[28] G. F. Gribakin and V. V. Flambaum, Phys. Rev. A 48, 546 (1993).

[29] W. Li, T. Pohl, J. M. Rost, S. T. Rittenhouse, H. R. Sadeghpour, J. Nipper, B. Butscher, J. B. Balewski, V. Bendkowsky, R. Löw, and T. Pfau, Science 334, 1110 (2011).

[30] See supplementary materials at [URL will be inserted by publisher].

[31] A. Gaj, A. T. Krupp, J. B. Balewski, R. Löw, S. Hofferberth, and T. Pfau, Nat. Commun. 5, 4546 (2014).

[32] J. Deiglmayr, H. Saßmannshausen, P. Pillet, and F. Merkt, Phys. Rev. Lett. 113, 193001 (2014). 